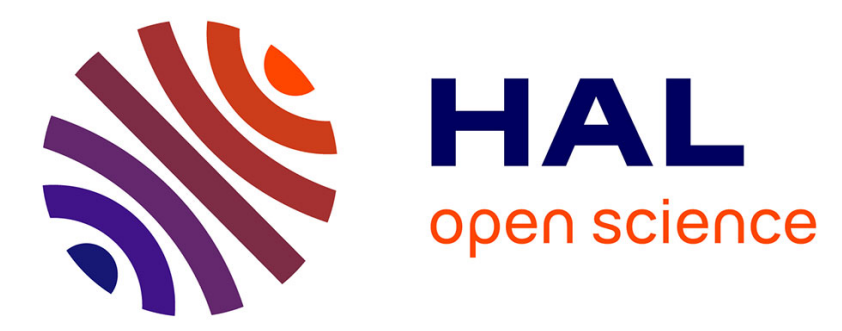

\title{
The substrate domain of BCAR1 is essential for anti-estrogen-resistant proliferation of human breast cancer cells
}

\author{
Arend Brinkman, Danielle Jong, Sietske Tuinman, Najat Azaouagh, Ton \\ Agthoven, Lambert C. J. Dorssers
}

\section{To cite this version:}

Arend Brinkman, Danielle Jong, Sietske Tuinman, Najat Azaouagh, Ton Agthoven, et al.. The substrate domain of BCAR1 is essential for anti-estrogen-resistant proliferation of human breast cancer cells. Breast Cancer Research and Treatment, 2009, 120 (2), pp.401-408. 10.1007/s10549-009-0403-4 . hal-00535363

\section{HAL Id: hal-00535363 \\ https://hal.science/hal-00535363}

Submitted on 11 Nov 2010

HAL is a multi-disciplinary open access archive for the deposit and dissemination of scientific research documents, whether they are published or not. The documents may come from teaching and research institutions in France or abroad, or from public or private research centers.
L'archive ouverte pluridisciplinaire HAL, est destinée au dépôt et à la diffusion de documents scientifiques de niveau recherche, publiés ou non, émanant des établissements d'enseignement et de recherche français ou étrangers, des laboratoires publics ou privés. 


\title{
The substrate domain of BCAR1 is essential for anti-estrogen- resistant proliferation of human breast cancer cells
}

\author{
Arend Brinkman · Danielle de Jong • \\ Sietske Tuinman · Najat Azaouagh . \\ Ton van Agthoven · Lambert C. J. Dorssers
}

Received: 25 March 2009/Accepted: 9 April 2009/Published online: 3 May 2009

(C) Springer Science+Business Media, LLC. 2009

\begin{abstract}
To unravel the mechanisms underlying failure of endocrine therapy of breast cancer, we have previously executed a functional genetic screen and identified the adaptor protein BCAR1 to be causative for tamoxifen resistance. As a consequence of the manifold of interactions with other proteins, we characterized the contribution of individual protein domains of BCAR1 to anti-estrogenresistant proliferation of human breast cancer cells. We took advantage of the observation that the closely related family member HEF1 was unable to support long-term anti-estrogen-resistant cell proliferation. Chimerical proteins containing defined domains of BCAR1 and HEF1 were evaluated for anti-estrogen-resistant growth. Exchange of the SH3 and $C$-terminal domains did not modify the capacity to support cell proliferation. Full support of antiestrogen resistant proliferation was observed for chimerical molecules containing the central part of BCAR1. The bi-partite SRC-binding site or the Serine-rich domain did not explain the differential capacity of BCAR1. These findings indicate that the differences between BCAR 1 and HEF1 with respect to support of anti-estrogen resistance reside in the substrate domain which contains multiple sites for tyrosine phosphorylation. The crucial interactions required for anti-estrogen resistance occur within the substrate domain of BCAR1. Further deciphering of these interactions may resolve the growth regulatory mechanism and provide an explanation for the observation that primary tumors with high levels of BCAR1 are likely to fail on
\end{abstract}

\footnotetext{
A. Brinkman · D. de Jong - S. Tuinman - N. Azaouagh ·

T. van Agthoven - L. C. J. Dorssers ( $\triangle)$

Department of Pathology, Josephine Nefkens Institute,

Erasmus MC, University Medical Centter Rotterdam,

Be432, P.O. Box 2040, 3000 CA Rotterdam, The Netherlands

e-mail: 1.dorssers@erasmusmc.nl
}

tamoxifen therapy. This information may also help to devise alternative personalized treatment strategies with improved outcome for breast cancer patients.

Keywords Endocrine therapy - Tamoxifen resistance . Fulvestrant · Chimerical proteins - Adaptor proteins

\section{Introduction}

Endocrine therapy of breast cancer is applied widely and has proven to be effective. Breast tumor development and recurrence can be reduced by long-term treatment with the estrogen antagonist tamoxifen [1]. In addition, tamoxifen and other endocrine treatment regimens have shown clinical benefit in advanced disease [2]. Tamoxifen competes with estrogen for the estrogen receptor (ER) $\alpha$ and interferes effectively with estrogen signaling. However, in most patients with advanced disease, the available endocrine treatments ultimately fail due to the development of therapy resistance. Despite the detailed insights in ER function, the mechanism of this general therapy failure is still poorly understood [3-7].

In our search for breast cancer anti-estrogen resistance $(B C A R)$ genes involved in the development of tamoxifen resistance, we have applied a functional genetic screen using retrovirus insertion mutagenesis. Estrogen-dependent human breast cancer cells (ZR-75-1) were infected with defective retrovirus and subsequently selected for growth in the presence of 4-hydroxy tamoxifen. Insertion of a retrovirus into the host cell genome can alter the expression of genes located in its proximity and individual cells may survive the selection when the altered gene confers tamoxifen resistance. The BCAR1 locus was the first retroviral integration site identified and demonstrated to 
contain a causative gene for the resistant phenotype $[8,9]$. Additional experimentation demonstrated that the BCARI gene was the human homologue of rat p130Cas, a prominent tyrosine phosphorylated protein in Src- and Crktransformed cells, and also confirmed the dominant role of $B C A R 1$ in tamoxifen-resistant cell growth [10-12]. Recently, BCARI has also been implicated in resistance to the chemotherapeutic drug adriamycin [13]. Furthermore, by analysis of patient specimens we have shown that patients with primary breast tumors containing high BCAR1 protein levels have a poor prognosis and a poor type of response to tamoxifen treatment [14-18].

BCAR1/p130Cas (hereafter referred to as BCAR1), exhibits a modular structure containing a Sarc homology 3 (SH3) domain, a Proline-rich region, a substrate region containing multiple YXXP phosporylation motifs, a Serine-rich domain, a Src Binding domain and a conserved $C$ terminus $[19,20]$. These domains are mostly conserved among the family members NEDD9 (HEF1/Cas-L), EFS (Sin) and HEPL [21]. As a consequence of the multiple protein interaction sites, BCAR1 serves as a docking molecule and has been implicated in many cellular processes, including cell transformation, integrin signaling, estrogen signaling, cell death, cytoskeletal rearrangements, migration, proliferation, force sensing, and bacterial infection [7, 12, 19, 20, 22-25]. The closely related family member NEDD9/HEF1/CAS-L (hereafter referred to as HEF1) is unable to substitute for BCAR1 in vivo, since p130Cas knock-out mice die in utero due to developmental cardiovascular defects and growth retardation [26]. HEF1 has been shown to participate in cell migration, cell cycle regulation and in melanoma metastasis [27-29].

How BCAR1 achieved tamoxifen-resistant tumor cell proliferation was not clear from our previous work, but was anticipated to involve SRC-BCAR1 complexes [7, 20, 23,
24]. SRC was shown to bind to the Src-binding site and to phosphorylate several YXXP motifs of BCAR1 [30-33]. These experiments have shown that BCAR1 phosphorylation may be important for some processes (cell migration), but not essential for anchorage-independent growth of SRC transformed fibroblasts [7, 23, 24, 34-36]. Another important finding was the functional association between the $C$-terminal ends of BCAR1 and BCAR3 (AND-34) [37-41], the latter also identified in our functional screen for tamoxifen resistance [42].

In this manuscript we describe the use of protein chimeras of BCAR1 and HEF1 to establish the contribution of individual protein domains to anti-estrogen-resistant proliferation of human breast cancer cells.

\section{Materials and methods}

Generation of variant constructs and chimera

The BCAR1 cDNA (AJ242987) and the HEF1 cDNA (GeneStorm clone L43821, Invitrogen, Breda, The Netherlands) were inserted into pCR2.1-TOPO vector plasmid (Invitrogen) between the KpnI and EcoRI sites. Restriction sites were removed or introduced using specific primers (Table 1) and the Quickchange Site-Directed Mutagenesis kit (Stratagene), and sequence verified as described previously [9]. In brief, a HindIII site was introduced at the end of the SH3 domain (nt position 302, leading to an amino acid change 62I->L), a SalI and HindIII site (nt 1334 and 1739, respectively) removed and a SalI site introduced at position $2120(668 \mathrm{H}->\mathrm{D})$ within BCAR1 (BCAR1a, Fig. 1). In HEF1, a SalI site was introduced (nt 2057) leading to $633 \mathrm{H}->\mathrm{D}$ (HEF1a). For exchange of

Table 1 Mutation primer sequences

\begin{tabular}{lll}
\hline Gene/modification & Forward $\left(5^{\prime} \rightarrow 3^{\prime}\right)$ & Reverse $\left(5^{\prime} \rightarrow 3^{\prime}\right)$ \\
\hline BCAR1/HindIII (302) & GGGAACCGCCTCAAGCTTTTGGTGGGCATG & CATGCCCACCAAAAGCTTGAGGCGGTTCCC \\
BCAR1/delHindIII (1739) & GCCCTGCATGCCAAACTTAGCCGGCAGCTG & CAGCTGCCGGCTAAGTTTGGCATGCAGGGC \\
BCAR1/SALI (2120) & GACTATGACTACGTCGACCTACAGGGGAAG & CTTCCCCTGTAGGTCGACGTAGTCATAGTC \\
BCAR1/delSALI (1334) & GGTGGCGTGGTGGACAGTGGTGTGTATGCG & CGCATACACACCACTGTCCACCACGCCACC \\
HEF1/SALI (2057) & GACTACGATTACGTCGACCTACAGGGTAAG & CTTACCCTGTAGGTCGACGTAATCGTAGTC \\
HEF1/SALI (1247) & GGCTCTCGGGACTTGGTCGACGGGATCAACCG & CGGTTGATCCCGTCGACCAAGTCCCGAGAGCC \\
ATG adaptor & AGCTTCAGGTGGTTCATGGTGTCCGGCGG & AATTCCGCCGGACACCATGAACCACCTGA \\
BCAR1/-135AA & GGCGGCCTGGGGCCCTAGGACCGGCAGCTG & CAGCTGCCGGTCCTAGGGCCCCAGGCCGCC \\
BCAR1/RPLA & CAGTCACGACCCCTAGCCTCACCCCCTAAG & CTTAGGGGGTGAGGCTAGGGGTCGTGACTG \\
BCAR1/FDF & GGATGGAGGACTTTGACTTCGTCGACCTACAGG & CCTGTAGGTCGACGAAGTCAAAGTCCTCCATCC \\
Flag-tag/BCAR1 & GCCGCCACCATGGATTACAAGGATGACGACGATAAGAACCACCTGAACGTGCTGGCCAA \\
Flag-tag/HEF1 & GCCGCCACCATGGATTACAAGGATGACGACGATAAGAAGTATAAGAATCTTATGGCAAGG \\
\hline
\end{tabular}




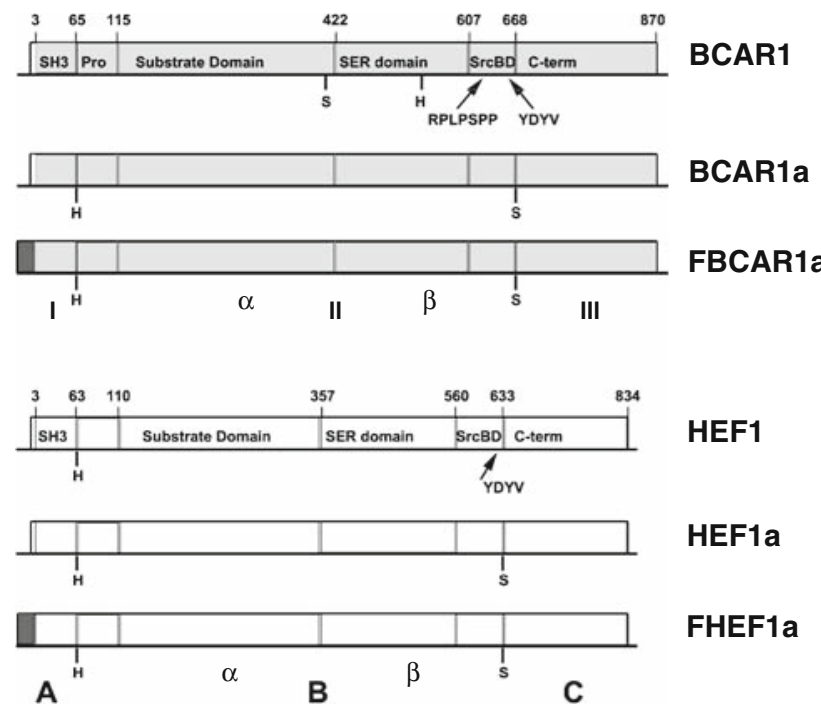

Fig. 1 Schematic representation of BCAR1 and HEF1. The structure including the SH3 domain, proline-rich region (Pro), Substrate domain, the serine-rich region (SER domain), the SRC binding domain (SrcBD) containing the SH3 binding site (RPLPSPP) and the substrate site (YDYV), and the $C$-terminal domain $(C$-term) of BCAR1 is shown. The homologous domains within HEF1 have been marked identically. Numbers represent amino acid positions. Restriction sites $(\mathrm{H}=$ HindIII, $\mathrm{S}=$ SalI $)$ used for the construction are indicated. Numbers and letters below the graphs describe the respective regions used for generation of the chimerical variants

the Ser-rich domain, an additional SalI site was introduced at nt position 1247 of HEF1, without altering the amino acid sequence. In addition, we have generated flagged versions of BCAR1a (FBCAR1a) and HEF1a (FHEF1a) by exchanging the $5^{\prime}$-upstream region including the initiation codon for the FLAG tag sequence (Table 1). For generation of a $N$-terminal deletion construct, the $\mathrm{SH} 3$ domain was removed by adding an initiation codon preceding the HindIII site at position 202 (ATG adaptor primer). For the $C$-terminal truncated version lacking 135 amino acids, a termination codon was introduced at the specific position (BCAR1/-135AA primer). The SRC SH3-domain binding sequence (RPLPSPP) was mutated to RPLASPP, and the phosphotyrosine substrate domain (YDYV) was modified to FDFV within BCAR1a using the primers detailed in Table 1 . The overall structure and relevant restriction sites used for generation of (chimerical) expression constructs are depicted in Fig. 1. Chimerical constructs were produced by ligation of the appropriate restriction fragments into the PCR2.1-TOPO plasmid. All resulting plasmids were sequence verified and tested for production of full length protein using the in vitro transcription-translation assay (ITT, Promega, Leiden, The Netherlands). Expression cassettes were excised with the unique cutting restriction enzymes XhoI and EcoRI and inserted in the LZRS-IRES-Neo vector [12], and the resulting expression vectors characterized by detailed restriction enzyme analyses.

Cell culture

The human ZR-75-1 breast cancer cells were maintained in RPMI medium supplemented with $10 \%$ bovine calf serum (R/BCS) and $1 \mathrm{nM}$ of $17 \beta$-estradiol as previously described $[9,43]$. Transfection of LZRS-derived expression constructs was performed using FuGENE 6 (Roche Diagnostics $\mathrm{GmbH}$, Mannheim, Germany) following the protocol of the supplier. Transfectants were selected using $1 \mathrm{mg} / \mathrm{ml}$ of G418 (Geneticin, Invitrogen) in complete medium and pooled cell populations were used for evaluation of antiestrogen resistance. Proliferation assays were performed by plating $0.7 \times 10^{6}$ cells in R/BCS medium supplemented with $100 \mathrm{nM}$ of ICI182,780 in triplicate. The culture medium was replaced twice a week. Cells were harvested using trypsin/EDTA at the indicated days, counted and reseeded at the original density as previously described [12]. Expression of the relevant proteins was verified by western blot analyses immediately before start of the assay or after the first passage.

Protein analyses

Cultured cells were trypsinized, rinsed with PBS buffer, sonicated for $10 \mathrm{~s}$ and lysed in $1 \% \mathrm{SDS}, 10 \mathrm{mM}$ Tris-HCl $\mathrm{pH} 7.5$ buffer for $10 \mathrm{~min}$ at $100^{\circ} \mathrm{C}$ [12]. Protein content in total cell extracts was determined using the BCA protein assay reagent (Pierce Chemical Co Rockford, IL). Polyacrylamide gels were loaded with equivalent amounts of protein $(3.3 \mu \mathrm{g})$, blotted onto membrane and subjected to western blot analysis with enhanced chemiluminescence detection as previously described [14, 17, 42]. Antibodies used to verify the products generated from the expression constructs were: mouse monoclonal anti-Cas (Transduction Laboratories, Lexington, KY), rabbit polyclonal Cas (N-17) and Cas (C-20), Cas-L/HEF1 (N-17) (Tebu-Bio, Santa Cruz Biotechnology Inc., Santa Cruz, CA), Rabbit anti-BCAR1 (329\#) [17], and anti-Flag M2 (Stratagene Europe, Amsterdam, The Netherlands).

\section{Results}

BCAR1 variants

The BCAR1 family member HEF1 exhibits a very similar modular protein structure and thus may exert a role in tamoxifen resistance in the ZR-75-1 breast cancer cell model comparable with BCAR1. ZR-75-1 cells are completely dependent on estrogen and are growth arrested in 
medium supplemented with anti-estrogen [12, 42]. In contrast to BCAR1, HEF1 appeared unable to support long-term proliferation of ZR-75-1 cells in the presence of OH-Tam or ICI182,780 in pilot experiments (not shown). These observations initiated the construction of chimerical molecules between BCAR1 and HEF1 by exchanging defined protein domains. It was anticipated that exchanging complete domains would not result in a compromised protein fold and thus would provide insight into the critical domains of BCAR1 required for anti-estrogen-dependent cell proliferation.

In order to exchange the $N$-terminal $\mathrm{SH} 3$ domain, the central domain containing many protein interaction sites, and the $C$-terminal domain, we introduced convenient restriction sites between these domains (Fig. 1). In the BCARI cDNA, the endogenous HindIII and SalI sites were removed without altering the amino acid sequence. A novel HindIII site was introduced at the end of the SH3 domain causing an amino acid change 62I- $>$ L (also a leucine at this position in HEF1), and a SalI site before the $C$-terminal domain leading to an exchange of an aspartic acid into a histidine on residue 668 (BCAR1a). Within the HEF1 cDNA, a SalI site was introduced before the $C$-terminal domain also causing an identical amino acid change $(633 \mathrm{D} \rightarrow \mathrm{H}, \mathrm{HEF} 1 \mathrm{a})$. Additionally, a Flag-tag was added at the NH2-terminus of both BCAR1a and HEF1a, to facilitate the detection of the recombinant proteins (Fig. 1). Using these restriction sites on the boundaries of the protein domains, fusion constructs were generated, and were verified by both DNA sequence analysis and in vitro translation assays. In addition to these chimerical constructs, a set of deletion and mutation variants of BCAR1 was generated to investigate the role of individual domains and binding sites. The $N$-terminal $\mathrm{SH} 3$ domain was removed (BCAR1 $\triangle \mathrm{SH} 3$ ) as well as the $C$-terminal 135 amino acids (BCAR1 $\Delta 135$ ), the latter implicated in protein-protein interaction with BCAR3 [38]. All validated expression constructs were inserted into the LZRS-IRESNeo expression vector and transfected into ZR-75-1 cells [12]. Pools of transfected cell clones were selected with G418, and evaluated for expression of the appropriate protein (Fig. 2). ZR-75-1 cells possessed low levels of endogenous BCAR1 and transfectants with the BCAR1 expression constructs contained increased levels of protein (Fig. 2a). HEF1 was barely detectable in the parental cells (not shown), while transfectants carrying the HEF1 expression construct carried two proteins (Fig. 2b) most likely related to differential phosphorylation [44].

Anti-estrogen-resistant cell proliferation

ZR-75-1 cells over-expressing either the wild-type BCAR1 or HEF1 were tested for their capacity to support growth of
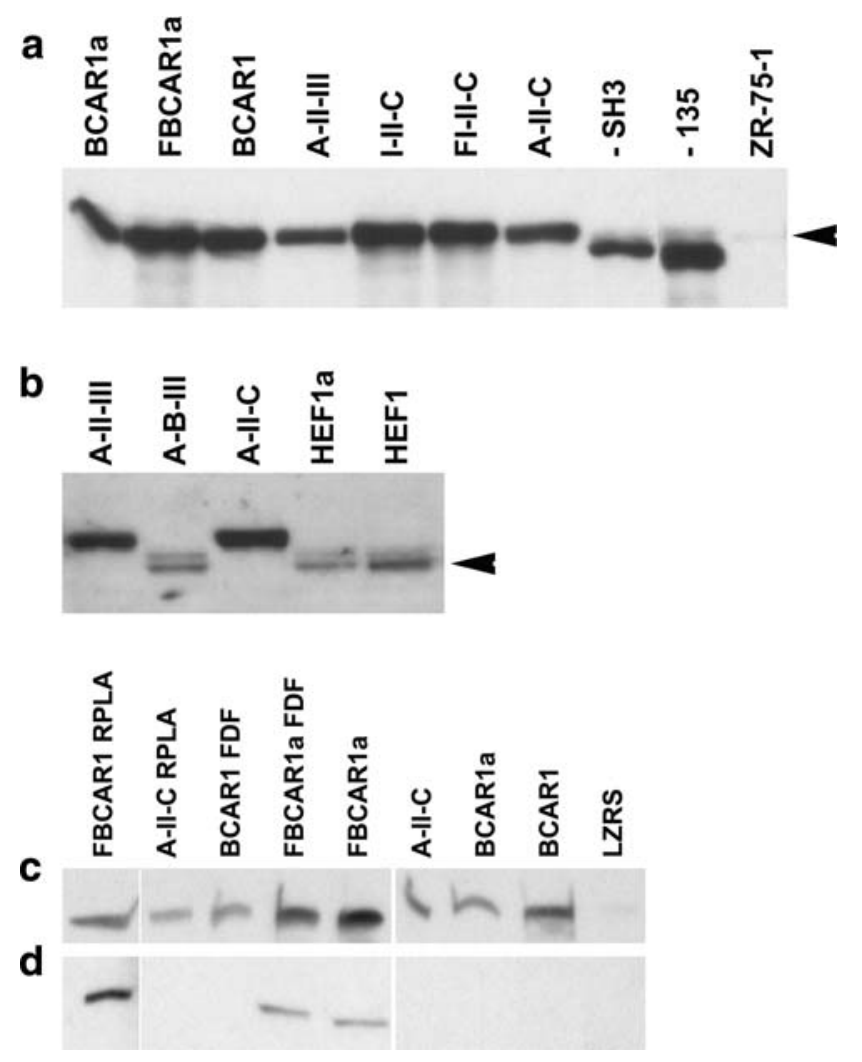

Fig. 2 Western blot analysis of BCAR1, HEF1 and variant proteins. Total cell lysates were separated by gel electrophoresis and blotted onto membrane. Proteins were visualized using antibodies directed against BCAR1 (\#329, a,c), HEF1 (b) or the Flag-tag (d). The arrows indicate the position of wild-type BCAR1 (a) or HEF1 (b)

the cells in the presence of anti-estrogen. In these experiments, we used the pure antagonist ICI182,780 as a rigorous selection system [12, 42]. Cumulative cells numbers relative to the starting numbers (Fig. 3) showed that BCAR1 over-expression (Fig. 2a) indeed conferred stable cell proliferation. Cells transfected with expression constructs containing wild-type HEF1 and showing overexpression of the protein (Fig. 2b), did increase in numbers during the first 2 weeks of culture, but then arrested. Control cells carrying the empty vector were rapidly growth arrested and became depleted in the culture. These results demonstrated that HEF1 does not support long-term anti-estrogen-resistant cell proliferation.

The various mutational variants of BCAR1 and HEF1 were tested in the same manner. Cells with verified overexpression of the different variants (Fig. 2) were plated at equal densities and cultured with ICI182,780 for several weeks. The conservative amino acid substitutions on residues 62 and 668 of BCAR1 as a consequence of the introduction of convenient restriction sites, did not affect the anti-estrogen-resistant growth capacity of cells transfected with this construct (BCAR1a, Fig. 3). Addition of the Flag-tag to the amino terminus of BCAR1a did neither 


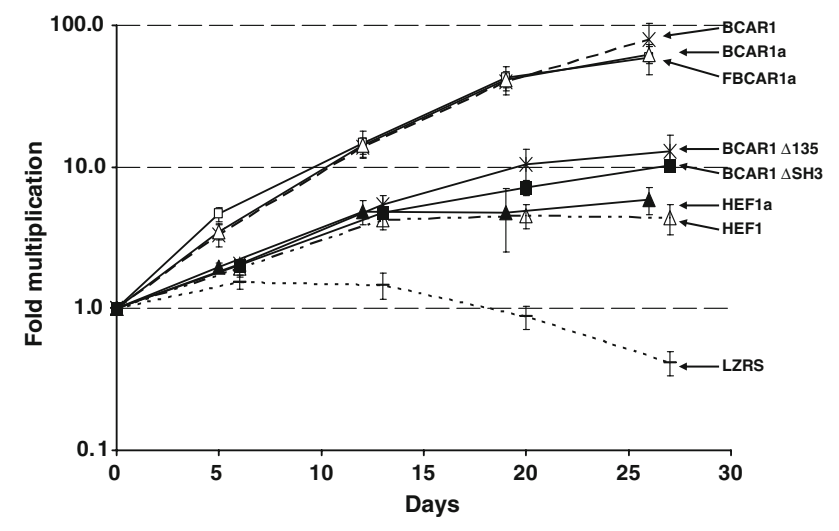

Fig. 3 Anti-estrogen-resistant cell proliferation assay of BCAR1, HEF1 and variants. Equal numbers of cells transfected with the indicated expression constructs were cultured in the presence of the anti-estrogen ICI182,780 (100 nM) in triplicate. Cells were harvested at the indicated time points, counted and re-seeded at the original density. Cumulative relative cell numbers and the SD are presented

affect its function (FBCAR1a). Similarly, the substitution of the residue 633 of HEF1 (HEF1a, Fig. 3) or the addition of the Flag-tag did not generate resistance in transfected cells producing these proteins. Cells expressing variants lacking either the $N$-terminal $\mathrm{SH} 3$ domain or the $C$-terminal 135 amino acids showed reduced growth when compared with wild-type BCAR1, but proliferated better than LZRS vector control or HEF1-transfected cells. It should be noted that cells expressing these truncated variants exhibited impaired binding to the substrate at later passages, which may have contributed to reduced cell recovery.

Several constructs expressing chimerical proteins were tested for anti-estrogen resistance in this proliferation assay. Exchange of the SH3 domain of BCAR1 (I, Fig. 1) for the HEF1 counterpart (A) showed no major effect on anti-estrogen-resistant growth of the cells (BCAR1 vs A-IIIII, Fig. 4). Furthermore, insertion of the SH3 domain from BCAR1 (I) into HEF1 constructs did not generate resistant cells (FI-B-C vs HEF1, Fig. 4). The $C$-terminal domains of BCAR1 (III) or HEF1 (C) neither made a distinction in the growth capacity of the cells in the presence of ICI182,780 (BCAR1 vs I-II-C or FI-II-C; HEF1 vs A-B-III, Fig. 4). In striking contrast, all constructs containing the central domain (II) of BCAR1 showed increased anti-estrogen resistant cell proliferation compared with constructs containing the corresponding domain (B) of HEF1 (Fig. 4). Insertion of HEF1 central part in BCAR1 (I-B-III, FI-B-III) caused loss of function, while introduction of the BCAR1 central domain in HEF1 (A-II-C) resulted in gain of function.

A major difference between HEF1 and BCAR1 is the partial conservation of the SRC-binding domain (Fig. 1). A proline rich sequence (RPLPSPP) and a tyrosine phosphorylation site (YDYV) in BCAR1 have been demonstrated to represent a binding site for the SRC kinase and to contribute to cellular functions [32, 45]. Individual mutations were introduced into BCAR1 to prevent the binding of SH3 domain-containing proteins to the proline-rich sequence motif and to prohibit phosphorylation of the specific tyrosine residues. Functional analyses of pools of transfectants showed that mutation of the RPLPSPP sequence, which disrupted interaction with SRC [45], did not significantly affect anti-estrogen-resistant cell proliferation (FBCAR1a vs FBCAR1a RPLA; A-II-C vs A-II-C RPLA, Fig. 5). The substitution of the tyrosine residues (YDYV->FDFV) did reduce the growth properties (FBCAR1a vs. FBCAR1a FDF; BCAR1a vs. BCAR1a FDF, Fig. 5), but also affected cell attachment following passage and could have led to reduced cell recovery.

In an attempt to further delineate the contribution of sub-domains, we have split the central domain in two separate fragments by using the Sal 1 site just preceding the Ser-rich domain of BCAR1 (Fig. 1). A Sal1 site was also introduced at the corresponding position of HEF1 and allowed for exchange of these sub-domains. A Flag-tagged BCAR1 construct containing the Ser-rich and SRC-binding domain $(\mathrm{B} \beta)$ of HEF1 retained most of the capacity to proliferate in the presence of anti-estrogen (FI-II $\alpha / \mathrm{B} \beta$-III, Fig. 6). A construct containing the Ser-rich and the SRCbinding domain (II $\beta$ ) of BCAR1 within the context of a Flagged version of HEF1 (FA-B $\alpha / \mathrm{II} \beta$-C, Fig. 6), was unable to restore anti-estrogen resistance.

\section{Discussion}

In this manuscript, we describe the contribution of individual protein domains of BCAR1 for the anti-estrogenresistant and estrogen-independent proliferation of breast cancer cells. It is important to note, that low levels of BCAR1 are present in ZR-75-1 cells and that overexpression is essential to obtain the tamoxifen-resistant phenotype. Furthermore, over-expression of the close family member HEF1 was shown insufficient to sustain long-term estrogen-independent cell growth. Since these proteins exhibit very similar domain structures (Fig. 1), we predicted that exchange of intact domains would retain the protein folding and thus allow for the evaluation of their contribution to estrogen-independent cell growth.

The SH3 domain mediates complex formation with proteins containing proline-rich sequence motifs [46]. The SH3 domains of BCAR1 and HEF1 exhibit extensive sequence homology (75\% identity) and have been found to bind to many different proteins, including FAK, which is involved in the phosporylation of BCAR1 [32]. $N$-terminal deletion of BCAR1, which eliminates the SH3 domains, did show partially impaired growth of the transfected cells. 
Fig. 4 Assay for anti-estrogen resistance of BCAR1 and HEF1 chimerical variants.

Proliferation assays with transfected cells were performed as described for Fig. 3. The overall structure of the chimera are indicated with parts derived from BCAR1 in grey, parts derived from HEF1 in white and the Flag-tag in dark grey

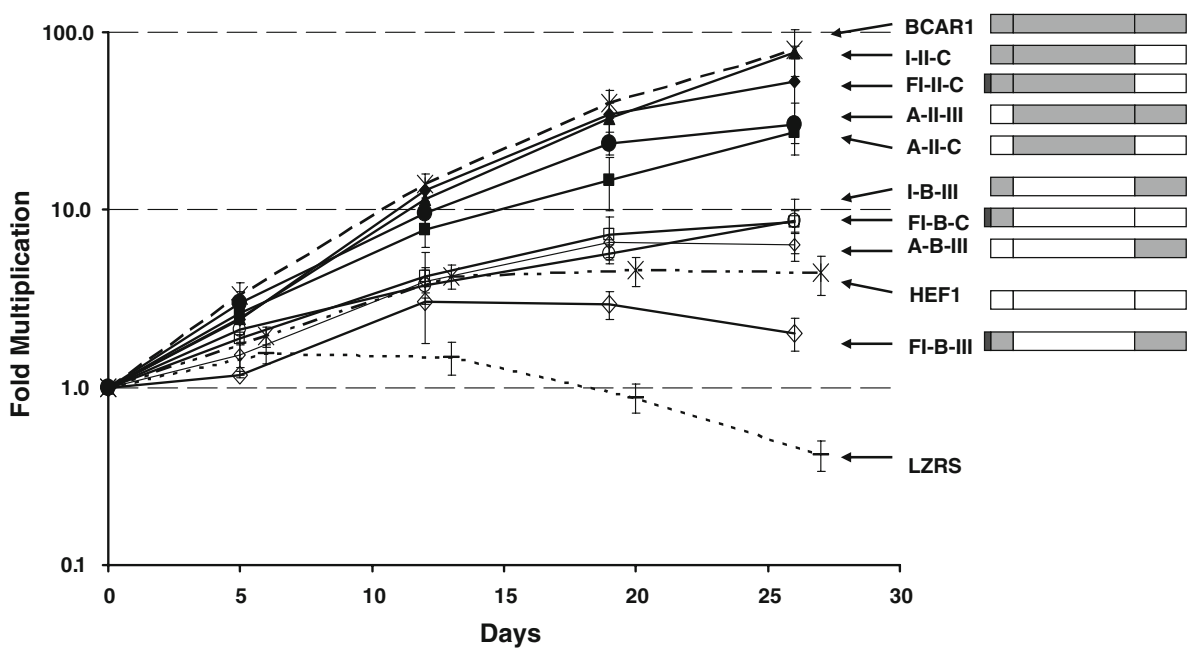

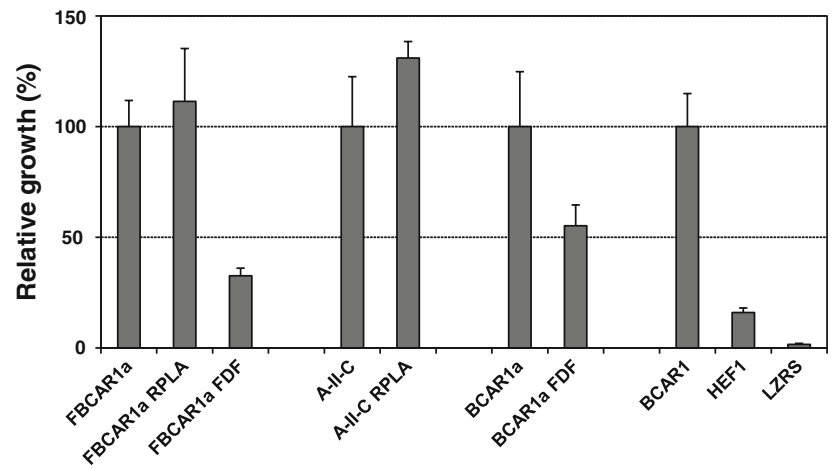

Fig. 5 Assay for anti-estrogen resistance of SRC-binding domain mutants. Assay was performed as described in Fig. 3. Cumulative cell numbers relative to the wild-type variant $(\%)$ at day 27 are given

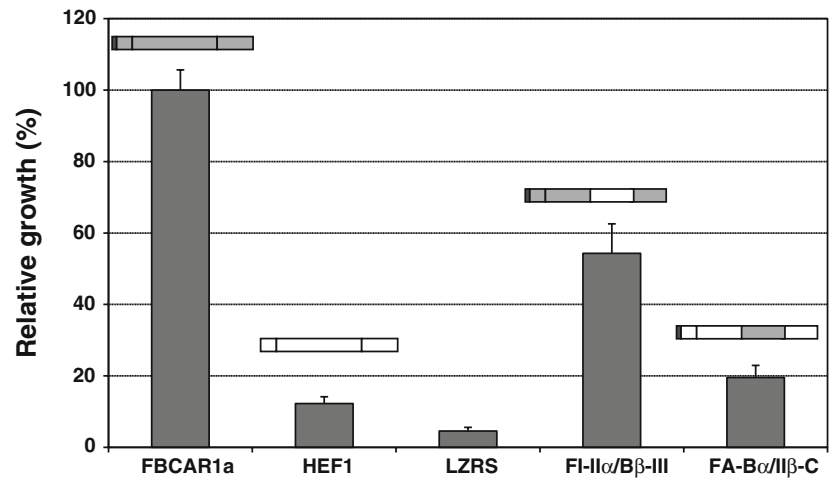

Fig. 6 Anti-estrogen-resistant cell proliferation assay of chimerical variants of the serine-rich region and the SRC-binding domain. Assay was performed as described in Fig. 5. Cumulative cell numbers relative to the wild-type (\%) at day 24 are presented for the variants. The structure of the chimera is as represented in Fig. 4

Furthermore we noticed reduced substratum binding following passage of these cells. The analysis of the chimerical variants showed that the origin of the $\mathrm{SH} 3$ domain was not important. The HEF1-derived SH3 domain supported anti-estrogen resistant cell proliferation nearly equally well as the wild-type domain of BCAR1. Apparently, no important differential interactions with binding partners exist between for the SH3 domains of HEF1 and BCAR1, which explain their differences in growth support in the presence of anti-estrogen.

The $C$-terminal parts of BCAR1 and HEF1 exhibit extensive sequence homology (54\% identity). This region of HEF1 contains a four helix bundle domain [47] and was shown to be important for filamentation of fungi [48], and both BCAR1 and HEF1 $C$-termini bind to BCAR3, another gene recovered from our functional screen for anti-estrogen resistance and predictive for tamoxifen resistance in breast cancer patients [38, 42, 47, 49]. Furthermore, p85 PI3 K was shown to bind to the $C$-terminus of p130Cas and to contribute to the growth of v-crk transformed cells [45]. Removal of the extreme 135 amino acids of BCAR1 implicated in BCAR3 binding did not fully abrogate growth. Furthermore, exchanging the complete $C$-terminus from BCAR1 by the domain from HEF1 was compatible with anti-estrogen resistant cell growth. These results suggest that interactions with the $C$-terminus do not explain the functional difference between BCAR1 and HEF1. Furthermore, BCAR3 binding is unlikely responsible for anti-estrogen resistance mediated by BCAR 1 because of the near absence of BCAR3 in wild type ZR-75-1 cells [42] and the absence of effects of BCAR3 knock-down in cells over-expressing BCAR1 (not shown).

The observation that replacement of the $N$ - and $C$-terminal domains of BCAR1 with the respective domains of HEF1 was fully compatible with growth (Fig. 4), suggested that the crucial elements must reside within the central domain. In agreement, insertion of the HEF1 central domain in BCAR1 caused loss of function while insertion of the BCAR1 central domain in HEF1 resulted in complete gain of function. The most prominent difference within the central domain is the partial absence of the 
bipartite SRC binding site in HEF1. While BCAR1 contains both the RPLPSPP SH3-recognition sequence and the YDYV substrate site, HEF1 possesses only the substrate site (Fig. 1). Mutation of the RPLPSPP site into RPLASPP in BCAR1 did not significantly impact growth of the cells, while alteration of the tyrosine residues in YDYV clearly impaired cell proliferation. In the latter experiments, cells slowly attached to the substratum after passage which may have impacted on cell survival and recovery. By introducing the Ser-rich domain and the SRC substrate site [50] of HEF1 within BCAR1, only minor effects were observed on cell proliferation in the presence of anti-estrogen. These results suggest that SRC binding and phosphorylation of the YDYV site do not have a major role in growth control for anti-estrogen resistance. This conclusion is supported by the observation that SRC kinase inhibitors (PP2) did not selectively affect growth of BCAR1-transfected cells in comparison with other anti-estrogen-resistant BCAR variants (data not shown). These cumulative data support the hypothesis that anti-estrogen resistance is mediated through the substrate domain of BCAR1. Within this domain, 15 YXXP motifs, which represent substrates for phosphorylation by tyrosine kinases and which may subsequently recruit different $\mathrm{SH} 2$-domain containing proteins, are conserved between rodents and humans [12]. Proteins binding to these motifs in the substrate domain of BCAR1 have been identified for different types of biological functions, including migration and regulation of the actin cytoskeleton [20, 36]. Despite extensive sequence differences within this substrate domain, 11 of these motifs have been retained in HEF1, although the spacing and sequence context may have changed (http:// www.ensembl.org/Homo_sapiens/Gene/Compara_Paralog/). Selective mutation of these individual tyrosine residues [36] may help to identify the set of crucial motifs required for anti-estrogen resistance. Proteomic approaches may also help to identify the crucial interacting proteins by comparison of chimerical variants with or without the capacity to support anti-estrogen-resistant cell growth.

In conclusion, our experiments have shown that retaining the conformation of the BCAR1 chimera allows the evaluation of larger parts of the protein for the capability to support anti-estrogen resistance. While truncated proteins were frequently affected in function, the exchange of homologous domains from HEF1 into BCAR1 often did not influence the function. Our experiments suggest that the differential effects of BCAR1 and HEF1 in long-term antiestrogen-resistant growth support predominantly reside within the central domain of these adaptor proteins. Several motifs within this domain may be a substrate for tyrosine kinases and their phosphorylation will recruit specific SH2domain-containing proteins and support the assembly of protein complexes driving cell growth. Further studies are required to pin down these critical interactions which may contribute to the poor response to tamoxifen treatment of patients with primary tumors containing high levels of BCAR1. Understanding these interactions will help to design personalized treatment strategies with improved outcome for breast cancer patients.

Acknowledgments We thank all current and past members of the molecular biology research lab for their stimulating support. This study was supported in part by grants of the Association for International Cancer Research (00-38), the Susan G. Komen Breast Cancer Foundation (BCTR0402594), and the Dutch Cancer Society.

\section{References}

1. Group Early Breast Cancer Trialists' Collaborative (2005) Effects of chemotherapy and hormonal therapy for early breast cancer on recurrence and 15-year survival: an overview of the randomised trials. Lancet 365:1687-1717

2. Jaiyesimi IA, Buzdar AU, Decker DA et al (1995) Use of tamoxifen for breast cancer: twenty-eight years later. J Clin Oncol 13:513-529

3. Ali S, Coombes RC (2002) Endocrine-responsive breast cancer and strategies for combating resistance. Nat Rev Cancer 2:101-112

4. Clarke R, Liu MC, Bouker KB et al (2003) Antiestrogen resistance in breast cancer and the role of estrogen receptor signaling. Oncogene 22:7316-7339

5. Osborne CK, Shou J, Massarweh S et al (2005) Crosstalk between estrogen receptor and growth factor receptor pathways as a cause for endocrine therapy resistance in breast cancer. Clin Cancer Res $11: 865 \mathrm{~s}-870 \mathrm{~s}$

6. Lewis JS, Jordan VC (2005) Selective estrogen receptor modulators (SERMs): mechanisms of anticarcinogenesis and drug resistance. Mutat Res 591:247-263

7. Riggins RB, Schrecengost RS, Guerrero MS et al (2007) Pathways to tamoxifen resistance. Cancer Lett 256:1-24

8. Dorssers LCJ, Van Agthoven T, Dekker A et al (1993) Induction of antiestrogen resistance in human breast cancer cells by random insertional mutagenesis using defective retroviruses: identification of bcar-1, a common integration site. Mol Endocrinol 7:870-878

9. Van Agthoven T, Veldscholte J, Smid M et al (2009) Functional screen identifies genes associated with human breast cancer progression. Breast Cancer Res Treat 114:23-30

10. Sakai R, Iwamatsu A, Hirano N et al (1994) A novel signaling molecule, p130, forms stable complexes in vivo with v-Crk and $\mathrm{v}-\mathrm{Src}$ in a tyrosine phosphorylation- dependent manner. EMBO J 13: $3748-3756$

11. Sakai R, Iwamatsu A, Hirano N et al (1994) Characterization, partial purification, and peptide sequencing of p130, the main phosphoprotein associated with v-Crk oncoprotein. J Biol Chem 269:32740-32746

12. Brinkman A, Van der Flier S, Kok EM et al (2000) BCAR1, a human homologue of the adapter protein p130Cas and antiestrogen resistance in breast cancer cells. J Natl Cancer Inst 92:112-120

13. Ta HQ, Thomas KS, Schrecengost RS et al (2008) A novel association between p130Cas and resistance to the chemotherapeutic drug adriamycin in human breast cancer cells. Cancer Res 68:8796-8804

14. Van der Flier S, Brinkman A, Look MP et al (2000) Bcar1/ p130Cas protein and primary breast cancer: prognosis and response to tamoxifen treatment. J Natl Cancer Inst 92:120-127

15. Van der Flier S, Van der Kwast TH, Claassen CJC et al (2001) Immunohistochemical study of the BCAR $1 / \mathrm{p} 130 \mathrm{Cas}$ protein in 
non-malignant and malignant human breast tissue. Int $\mathrm{J}$ Biol Markers 16:172-178

16. Van der Flier S, Chan CMW, Brinkman A et al (2000) BCAR1/ p130Cas expression in untreated and acquired tamoxifen-resistant human breast carcinomas. Int J Cancer 89:465-468

17. Grebenchtchikov N, Brinkman A, Van Broekhoven SPJ et al (2004) Development of an ELISA for measurement of BCAR1 protein in human breast cancer tissue. Clin Chem 50:1356-1363

18. Dorssers LCJ, Grebenchtchikov N, Brinkman A et al (2004) The prognostic value of BCAR1 in patients with primary breast cancer. Clin Cancer Res 10:6194-6202

19. O’Neill GM, Fashena SJ, Golemis EA (2000) Integrin signalling: a new cas $(t)$ of characters enters the stage. Trends Cell Biol 10: $111-119$

20. Bouton AH, Riggins RB, Bruce-Staskal PJ (2001) Functions of the adapter protein Cas: signal convergence and the determination of cellular responses. Oncogene 20:6448-6458

21. Singh MK, Dadke D, Nicolas E et al (2008) A novel Cas family member, HEPL, regulates FAK and cell spreading. Mol Biol Cell 19:1627-1636

22. Cabodi S, Moro L, Baj G et al (2004) p130Cas interacts with estrogen receptor alpha and modulates non-genomic estrogen signaling in breast cancer cells. J Cell Sci 117:1603-1611

23. Defilippi P, Di Stefano P, Cabodi S (2006) p130Cas: a versatile scaffold in signaling networks. Trends Cell Biol 16:257-263

24. Riggins RB, Thomas KS, Ta HQ et al (2006) Physical and functional interactions between Cas and c-Src induce tamoxifen resistance of breast cancer cells through pathways involving epidermal growth factor receptor and signal transducer and activator of transcription 5b. Cancer Res 66:7007-7015

25. Cabodi S, Tinnirello A, Di Stefano P et al (2006) p130Cas as a new regulator of mammary epithelial cell proliferation, survival, and HER2-neu oncogene-dependent breast tumorigenesis. Cancer Res 66:4672-4680

26. Honda H, Oda H, Nakamoto $T$ et al (1998) Cardiovascular anomaly, impaired actin bundling and resistance to Src- induced transformation in mice lacking p130Cas. Nat Genet 19:361-365

27. Kim M, Gans JD, Nogueira C et al (2006) Comparative oncogenomics identifies NEDD9 as a melanoma metastasis gene. Cell 125:1269-1281

28. O'Neill GM, Seo S, Serebriiskii IG et al (2007) A new central scaffold for metastasis: parsing HEF1/Cas-L/NEDD9. Cancer Res 67:8975-8979

29. Singh M, Cowell L, Seo S et al (2007) Molecular basis for HEF1/ NEDD9/Cas-L action as a multifunctional co-ordinator of invasion, apoptosis and cell cycle. Cell Biochem Biophys 48:54-72

30. Nakamoto T, Sakai R, Ozawa K et al (1996) Direct binding of $C$ terminal region of $130^{\mathrm{Cas}}$ to $\mathrm{SH} 2$ and $\mathrm{SH} 3$ domains of $\mathrm{Src}$ kinase. J Biol Chem 271:8959-8965

31. Burnham MR, Bruce-Staskal PJ, Harte MT et al (2000) Regulation of c-SRC activity and function by the adapter protein CAS. Mol Cell Biol 20:5865-5878

32. Ruest PJ, Shin NY, Polte TR et al (2001) Mechanisms of CAS substrate domain tyrosine phosphorylation by FAK and Src. Mol Cell Biol 21:7641-7652

33. Sharma A, Mayer BJ (2008) Phosphorylation of p130Cas initiates Rac activation and membrane ruffling. BMC Cell Biol 9:50

34. Shen Y, Jia Z, Nagele RG et al (2006) SRC uses Cas to suppress Fhl1 in order to promote nonanchored growth and migration of tumor cells. Cancer Res 66:1543-1552
35. Shen Y, Khusial PR, Li X et al (2007) SRC utilizes Cas to block gap junctional communication mediated by connexin43. J Biol Chem 282:18914-18921

36. Patwardhan P, Shen Y, Goldberg GS et al (2006) Individual Cas phosphorylation sites are dispensable for processive phosphorylation by $\mathrm{Src}$ and anchorage-independent cell growth. J Biol Chem 281:20689-20697

37. Cai D, Clayton LK, Smolyar A et al (1999) AND-34, a novel p130Cas-binding thymic stromal cell protein regulated by adhesion and inflammatory cytokines. J Immunol 163:2104-2112

38. Cai D, Iyer A, Felekkis KN et al (2003) AND-34/BCAR3, a GDP exchange factor whose overexpression confers antiestrogen resistance, activates Rac, PAK1, and the cyclin D1 promoter. Cancer Res 63:6802-6808

39. Gotoh T, Cai D, Tian X et al (2000) p130Cas regulates the activity of AND-34, a novel Ral, Rap1, and R-Ras guanine nucleotide exchange factor. J Biol Chem 275:30118-30123

40. Riggins RB, Quilliam LA, Bouton AH (2003) Synergistic promotion of c-Src activation and cell migration by Cas and AND34/BCAR3. J Biol Chem 278:28264-28273

41. Schrecengost RS, Riggins RB, Thomas KS et al (2007) Breast cancer antiestrogen resistance-3 expression regulates breast cancer cell migration through promotion of p130Cas membrane localization and membrane ruffling. Cancer Res 67:6174-6182

42. Van Agthoven T, Van Agthoven TLA, Dekker A et al (1998) Identification of BCAR3 by a random search for genes involved in antiestrogen resistance of human breast cancer cells. EMBO J 17:2799-2808

43. Van Agthoven T, Van Agthoven TLA, Portengen H et al (1992) Ectopic expression of epidermal growth factor receptors induces hormone independence in ZR-75-1 human breast cancer cells. Cancer Res 52:5082-5088

44. Law SF, Zhang YZ, Klein-Szanto AJP et al (1998) Cell cycleregulated processing of HEF1 to multiple protein forms differentially targeted to multiple subcellular compartments. Mol Cell Biol 18:3540-3551

45. Riggins RB, DeBerry RM, Toosarvandani MD et al (2003) SrcDependent Association of Cas and p85 Phosphatidylinositol 3'-Kinase in v-crk-Transformed Cells. Mol Cancer Res 1:428-437

46. Pawson $\mathrm{T}$ (1995) Protein modules and signalling networks. Nature 373:573-580

47. Garron ML, Arsenieva D, Zhong J et al (2009) Structural insights into the association between BCAR3 and Cas family members, an atypical complex implicated in Anti-oestrogen resistance. J Mol Biol 386:190-203

48. Law SF, Estojak J, Wang BL et al (1996) Human enhancer of filamentation 1, a novel p130 ${ }^{\text {cas }}$-like docking protein, associates with focal adhesion kinase and induces pseudohyphal growth in Saccharomyces cerevisiae. Mol Cell Biol 16:3327-3337

49. Van Agthoven T, Sieuwerts AM, Meijer-Van Gelder ME et al (2009) Relevance of breast cancer antiestrogen resistance genes in human breast cancer progression and tamoxifen resistance. J Clin Oncol 27:542-549

50. Nasertorabi F, Tars K, Becherer K et al (2006) Molecular basis for regulation of Src by the docking protein p130Cas. J Mol Recognit 19:30-38 